6 Thanacoody HK. Chronic valproic acid intoxication: reversal by naloxone. Emerg Med J 2007;24:677-8.

7 Alberto G, Erikson T, Popiel R, Narayanan M, Hryhorczuk D. Central nervous system manifestations of a valproic acid overdose responsive to naloxone. Ann Emerg Med 1989;18:889-91.

8 Eze E, Workeman M, Donley B. Hyperammonaemia and coma developed in woman treated with valproic acid for affective disorder. Psychiatr Serv 1998;49:1358-9.

9 Bohmer T, Bøem A, Hoymork C. Valproate-induced hyperammonemic encephalopathy rapidly induced by i.v. carnitine and glucose/thiamine. Scand J Gastroenterol 2010;45:762-3.
10 Dingledine R, Iversen LL, Breuker E. Naloxone as a GABA antagonist: evidence from iontopheric, receptor binding and convulsant studies. Eur ] Pharmacol 1978:47:19-27.

Address for correspondence: Dr Lara Camilleri, Mater Dei Hospital, Mazeltov, Dahlet Guzeppi Stagno, Marsaxlokk MXK1631, Malta.

Email: lara.camilleri@gov.mt

\title{
Lesson of the month 2: An unusual cause of depression in an older man: Cushing's syndrome resulting from metastatic small cell cancer of the prostate
}

\author{
Authors: Paul Kleinig ${ }^{\mathrm{A}, *}$ and Patrick Russell ${ }^{\mathrm{B}, *}$
}

This article describes the case of an older patient whose main presenting problem was depression. A constellation of clinical and biochemical features led to a search for medical causes of his low mood, which uncovered ectopic adrenocorticotropic hormone (ACTH) secretion. Post-mortem examination showed that the source of ACTH was small cell neuroendocrine carcinoma of the prostate, and that the patient had died from the sequelae of profound immune suppression.

KEYWORDS: Autopsy, ACTH syndrome, ectopic, clinicopathological conference, carcinoma, small cell, depression, prostatic neoplasms

\section{Case presentation}

An 84-year-old man presented to his GP with several weeks of fatigue, back pain and anorexia. Blood tests showed moderately elevated prostate-specific antigen (PSA) and creatinine. He had a history of locally invasive, but asymptomatic, prostate cancer. Ultrasound examination showed bilateral hydroureter and a prostate volume of $46 \mathrm{~mL}$. He underwent ureteric stenting, but his postoperative course was complicated by hypokalaemia, depressed mood and back pain. Magnetic resonance imaging of the back revealed no cause of his pain, and he was discharged.

Authors: ${ }^{\text {A }}$ palliative care consultant, Southern Adelaide Local

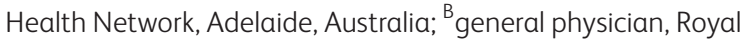
Adelaide Hospital, Adelaide, Australia; "authors contributed equally
One week later, the patient presented to the emergency department of a large public hospital with back pain radiating to his left buttock; mild bilateral proximal weakness was present, with no other neurological deficit. His depressed mood had worsened markedly and he was losing weight. He was again hypokalaemic, with associated hypertension and also hyperglycaemia, with a metabolic alkalosis. Regular psychiatric review confirmed some initial improvement in his mood following the start of treatment with mirtazapine. A computed tomography scan of pelvis showed a pelvic mass centred on the prostate with a maximal diameter of $105 \mathrm{~mm}$ and a calculated volume of $657 \mathrm{~mL}$ (Fig 1), with scattered pulmonary nodules and a heterogeneous appearance to his liver. His psychiatric and metabolic state deteriorated further. Testing found elevated serum cortisol $(7,220 \mathrm{nmol} / \mathrm{L}$ [normal range 200-700 nmol/L]), unsuppressed by low-dose dexamethasone $(10,400 \mathrm{nmol} / \mathrm{L}$ [normal $<50 \mathrm{nmol} / \mathrm{L}])$. His 24 -hour urinary free cortisol (>14,088 nmol [normal 55-250 nmol]) and plasma adrenocorticotropic hormone (ACTH) levels ( $87 \mathrm{pmol} / \mathrm{L}$ [normal 2-12 pmol/L]) were also abnormal, consistent with a diagnosis of Cushing's syndrome. His condition deteriorated rapidly and he died of sepsis, despite treatment with broad-spectrum antibiotics. He died after 3 weeks in the second hospital and only 6 weeks after admission under his private urologist.

Autopsy was performed to clarify the histology of the pelvic mass and the source of the ectopic ACTH. Post-mortem examination revealed a large mass with invasion of the pelvic sidewalls and bilateral ureters. Histologic examination revealed an invasive prostatic acinar adenocarcinoma with areas of small cell neuroendocrine carcinoma (Fig 2). There were innumerable metastatic tumour deposits within the liver, lung nodules 


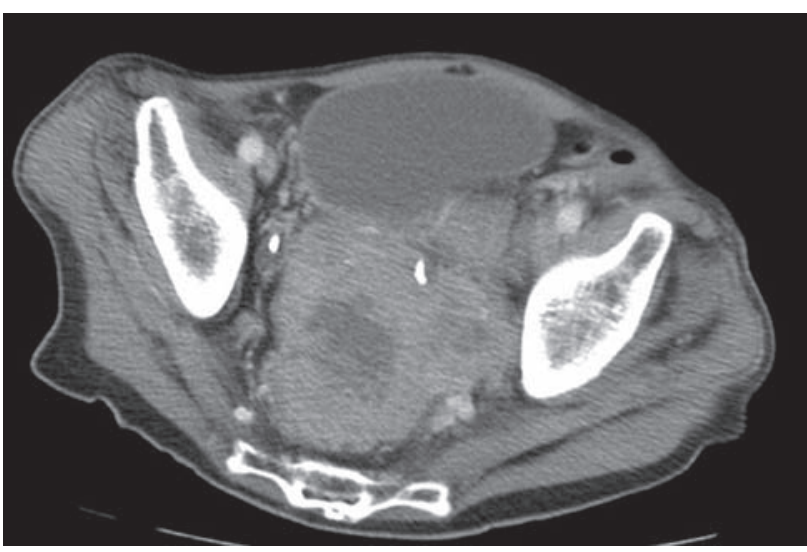

Fig 1. Axial computed tomography scan at the level of the pelvis showing a large irregular partially hypodense mass centred on the prostate. Ureteric stents are visible as small hyperdense lesions abutting the anterior wall of the mass.

and an adrenal lesion, which were all found to be metastatic neuroendocrine small cell cancer.

Immunoperoxidase studies confirmed the neuroendocrine nature of the small cell carcinoma, with tumour cells showing strong positive labelling for neuroendocrine markers (ie chromogranin, CD56 and synaptophysin). The conventional acinar adenocarcinoma showed labelling towards prostatic markers: PSA and prostatic acid phosphatase (PACP). There was no labelling of either tumour components for antibodies to TTF-1, and neither was any labelling demonstrated towards ACTH, although the ectopic ACTH was presumed to have originated here; no immunoperoxidase labelling was available for corticotropinreleasing hormone. The small cell cancer was thought to have dedifferentiated from conventional adenocarcinoma.

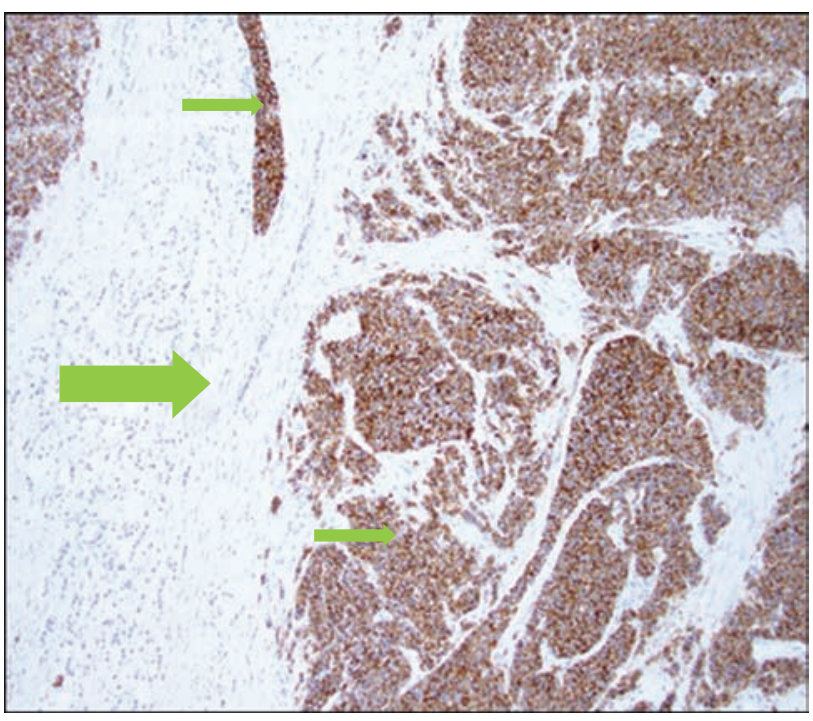

Fig 2. Histology with immunohistochemical staining of the post-mortem prostate showing neuroendocrine carcinoma (small arrows) with strong labelling for synaptophysin and conventional adenocarcinoma (large arrow) with absent staining.
Bronchopneumonia secondary to invasive aspergillus infection was the ultimate cause of death.

\section{Discussion}

The first described case of small cell cancer of the prostate was by Wenk in 1977. ' Carcinoma of the prostate with dominant small cell histology is rare, accounting for $0.5-2 \%$ of prostatic malignancies. ${ }^{2}$ In most cases, the tumour arises from known adenocarcinoma, usually high grade. ${ }^{2,3}$ Small cell cancer of the prostate can dedifferentiate from adenocarcinoma cells, neuroendocrine populations within the adenocarcinoma might develop malignant potential, or it might be that both cell lineages differentiate from a single totipotent stem cell. ${ }^{2-4}$ Androgen deprivation is likely a key causative factor in these cases, either by promoting the dedifferentiation of adenocarcinoma cells, or by selectively promoting the growth of neuroendocrine cells, which lack an androgen receptor. ${ }^{4}$ There are several well-described cases of ectopic ACTH production from small cell carcinoma of the prostate.

Ectopic ACTH secretion is also rare. Most ectopic ACTH secretion is caused by pulmonary neuroendocrine tumours. ${ }^{5}$ The definitive management of the syndrome is control of the tumour, but in cases of highly aggressive cancer, this is often not possible without a locally curative therapy. ${ }^{6}$ Indeed, case series of ectopic ACTH because of small cell lung cancer show poor response rates to cytotoxic chemotherapy, high rates of complication (predominantly infectious) and overall poor prognosis. ${ }^{7}$ There is a possibility of treatment extending life if hypercortisolaemia and the tumour can be controlled concurrently. ${ }^{8}$

The clinical picture presented by our patient was typical for ectopic ACTH secretion: hypertension, muscle weakness and hypokalaemia appear in the majority. Approximately $50 \%$ of patients will develop psychiatric symptoms, infections (often opportunistic) or hyperglycaemia. ${ }^{7}$ Neuropsychiatric disturbances in states of glucocorticoid excess are felt to be a product of the detrimental effect of glucocorticoids on brain structure and function through diverse and incompletely understood mechanisms. ${ }^{9}$ Depression in older patients can be more complex than in younger patients, as disease processes and exposure to medications known to cause depression become more prevalent with advancing age. ${ }^{10}$

Four main learning points are to be gained from our case. First, the most salient clinical feature was a rapid onset of depression progressing to psychosis with profound hypoactive delirium. The clinical picture prompted a thorough search for an organic aetiology. Second, the persistence of hypokalaemia despite oral replacement and without clear precipitants prompted a deeper investigation. Third, neither the visceral metastases nor aggressive growth of the pelvic mass noted on imaging were typical of prostatic adenocarcinoma. Fourth, an incomplete diagnosis at death was the precipitant for a post-mortem examination. The autopsy findings were beneficial to the patient's family and treating team. The case was discussed at a regular teaching meeting at a large tertiary hospital and, thus, was beneficial to a wide medical audience.

\section{Conclusion}

This case highlights the importance of multidisciplinary evaluation of clinical cases both ante-mortem and post-mortem, and is a fine example of how autopsy findings can be used to benefit a wide audience. 


\section{Consent to publish}

Consent to publish was obtained from the patient's next of kin.

\section{References}

1 Wenk RE, Bhagavan BS, Levy R, Miller D, Weissburger W. Ectopic ACTH, prostatic oat cell carcinoma and marked hypernatremia. Cancer 1977;:40:773-8.

2 Wang L, Davidson DD, Montironi R et al. Small cell carcinoma of the prostate: molecular basis and clinical implications. Histol Histopathol 2015;30:413-24.

3 Sargos P, Ferretti L, Gross-Goupil M et al. Characterization of prostate neuroendocrine cancers and therapeutic management: a literature review. Prostate Cancer Prostatic Dis 2014;17:220-6.

4 Sun Y, Niu J, Huang J. Neuroendocrine differentiation in prostate cancer. Am J Transl Res 2009;1:148-62.

5 Ilias I, Torpy DJ, Pacak K et al. Cushing's syndrome due to ectopic corticotropin secretion: twenty years' experience at the national institutes of health. J Clin Endocrinol Metab 2005;90:4955-62.

6 Aniszewski JP, Young WF, Thompson GB, Grant CS, van Heerden JA. Cushing syndrome due to ectopic adrenocorticotropic hormone secretion. World J Surg 2001;25:934-40.
7 Dimopoulos MA, Fernandez JF, Samaan NA, Holoye PY, Vassilopoulou-Sellin R. Paraneoplastic Cushing's syndrome as an adverse prognostic factor in patients who die early in small cell lung cancer. Cancer 1992;69:66-71.

8 Collichio FA, Woolf PD, Brower M. Management of patients with small cell carcinoma and the syndrome of ectopic corticotropin secretion. Cancer 1994;73:1361-7.

9 Pivonello R, Simeoli C, DeMartino MC et al. Neuropsychiatric disorders in Cushing's syndrome. Front Neurosci 2015;9:1-6.

10 Morichi V, Dell'Aquilla G, Trotta F et al. Diagnosing and treating depression in older and oldest old. Curr Pharm Des 2015;21:1690-8.

Address for correspondence: Dr Paul Kleinig, Laurel Hospice, Level 7 Rehabilitation, Aged Care and Palliative Care Building, Flinders Medical Centre, Flinders Drive, Bedford Park, South Australia, Australia, 5041.

Email: Paul.kleinig@sa.gov.au 